\title{
On The Physical Existence of The Zeta Reticuli Moving Group: A Chemical Composition Analysis
}

\author{
Letícia D. Ferreira ${ }^{1}$, Gustavo F. Porto de Mello ${ }^{1}$, and Licio da Silva ${ }^{2}$ \\ ${ }^{1}$ Universidade do Federal do Rio de Janeiro, Observatório do Valongo, \\ Ladeira do Pedro Antônio 43, Rio de Janeiro, RJ, Brazil. \\ email: leticia@astro.ufrj.br, gustavo@astro.ufrj.br \\ ${ }^{2}$ Observatório Nacional, \\ Rua Gen. José Cristino 77, São Cristovão, Rio de Janeiro, RJ, Brazil \\ email: licio@on.br
}

\begin{abstract}
We report the spectroscopic analysis of six kinematical members of the Zeta Reticuli Moving Group, one of them for the first time. We confirm the existence of the Group by establishing a common abundance pattern for four kinematical members. High resolution spectra yielded abundances of $\mathrm{Si}, \mathrm{Ca}, \mathrm{Fe}, \mathrm{Ni}$ and $\mathrm{Ba}$, and others. Effective temperatures were derived from the excitation \& ionization equilibria of Fe lines of four stars. For these, and the remaining two members, temperatures were derived from colors and the fitting of theoretical spectra to the $\mathrm{H} \alpha$ line, and ages and masses were estimated from theoretical HR diagrams. We suggest that the Group is physical being metal-poor and $\sim 6$ Gyr old.
\end{abstract}

Keywords. techniques: spectroscopic, stars: abundances, stars: kinematics, Galaxy: evolution.

\section{Introduction}

Stellar Kinematic Groups (SKGs) are assemblages of stars which share approximately the same vectors of Galactic space velocity. Presumably they constitute a link between gravitationally bound systems, such as the open clusters, and the field stars, and they are supposed to share the same features of these systems, as coeval age and chemical composition. The rarity of old kinematic groups bears witness to the processes that tear them apart, probably encounters with massive objects, such spiral arms and molecular clouds, in time scales of a billion years or less. Therefore, the majority of these groups must be young but some relatively old groups have already been considered as is the case of the Zeta Reticuli group (del Peloso et al. 2000).

\section{Methodology}

We report a detailed spectroscopic analysis of four objects of this group (with FEROS data - 2001), beyond two new candidates selected kinematically (with OPD/LNA data 2005). The spectroscopic parameters were determined from the model atmosphere analysis (NMARCS) of nearly 100 lines of Fe I and $\sim 10$ lines of Fe II, through excitation/ ionization equilibria. Effective temperature was determined by three different constraints: excitation \& ionization equilibria of Fe lines, photometry and the modeling of the $\mathrm{H} \alpha$ profile, whenever possible. The chemical abundance of each element ( $\mathrm{Ca}, \mathrm{Ba}, \mathrm{Sc}, \mathrm{Y}, \mathrm{Ti}$, $\mathrm{V}$, Ce, $\mathrm{Cr}, \mathrm{Mn}, \mathrm{Fe}, \mathrm{Co}, \mathrm{Ni}$ e $\mathrm{Si}$ ) was determined by a program that uses the atmosphere model derived for star, in set with the measure of the equivalent widths gotten for each element. 


\section{Results}

For HD158614, we obtained $\mathrm{T}_{\text {eff }}$ from the $\mathrm{H} \alpha$ modelling. This temperature was derived by fitting the observed wings of $\mathrm{H} \alpha$, using the automated procedure described in Lyra \& Porto de Mello (2005), and we found a $\mathrm{T}_{\text {eff }}=5573 \pm 50 \mathrm{~K}$. The other objects have their $\mathrm{H} \alpha$ temperatures derived from previous works. We constructed the theoretical HR diagram for the group according to Yi et al. (2003) with the new atmospheric parameters found for the group members, using HIPPARCOS parallaxes (fig. 1). Evolutionarily, we attach to the group a $\sim 6 \mathrm{Gyr}$, considering the associated errors $\left[\sigma\left(\mathrm{T}_{\text {effs }}\right) \sim 30 \mathrm{~K}, \sigma(\xi) \sim 0.04 \mathrm{kms}^{-1}\right.$, $\sigma(\log \mathrm{g}) \sim 0.13 \mathrm{dex}, \sigma(\mathrm{Fe} / \mathrm{H}) \sim 0.05 \mathrm{dex}]$.

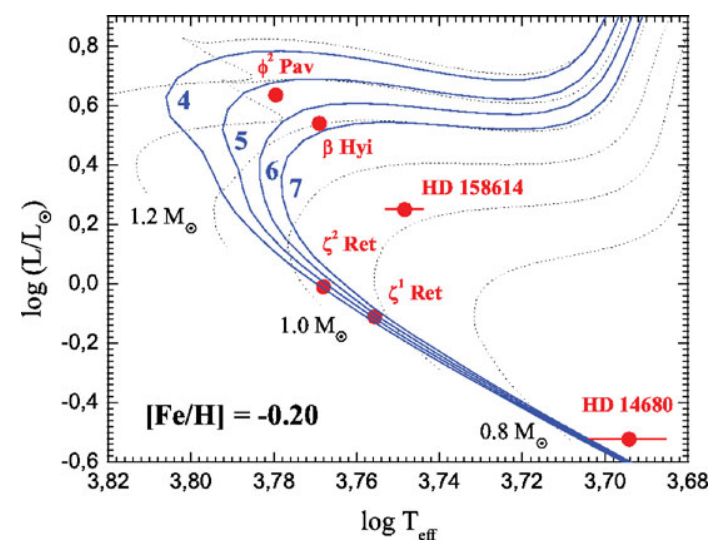

Figure 1. H-R diagram of the probable members of the $\zeta$ Ret moving group. The solid lines, numbered 4 to 7 , are the Yale isochrones for the metacility -0.20 dex.

\section{Conclusions and Perspectives}

The chemical analysis demonstrates that the group members define a metal-poor SKG at $[\mathrm{Fe} / \mathrm{H}]=-0,20$, with the exception of HD 158614 which does not seem to belong chemically to the group. Alternatively, HD 14680 presented a good chemical agreement with the group: yet an evolutionary analysis is not possible for this star owing to its very low mass, as it lies very close to its ZAMS location. The kinematical analysis shows that this star appears a little distant from the group kinematical core. Anyway, a better conclusion on the kinematics of this star would only be possible with a search for a larger sample of candidates, to more consistently evaluate its kinematical dispersion. An interesting conflict appears for $\zeta^{1}$ Ret. Del Peloso et al. (2000) suggest that these stars show a higher chromospheric flux than expected for its age. A possible solution to this problem may be the analysis of the chromospheric flux of $\zeta^{1}$ Ret through other chromospheric indicators, along with the other solar-type group members.

\section{References}

del Peloso, E. F., da Silva, L., \& Porto de Mello, G. F.2000, A\&A, 233, 358

Porto de Mello, G. F. 1996, Astrobiology, 6, 308

Lyra, W. \& Porto de Mello, G. F. 2005, A\&A, 329, 338

Yi, S. K., Kim, Y., \& Demarque, P. 2003, ApJS, 144, 259 